This item was submitted to Loughborough's Research Repository by the author.

Items in Figshare are protected by copyright, with all rights reserved, unless otherwise indicated.

\title{
Newtonian mechanics in scale of minutia
}

PLEASE CITE THE PUBLISHED VERSION

PUBLISHER

Professional Engineering Publishing / @ IMECHE

VERSION

VoR (Version of Record)

LICENCE

CC BY-NC-ND 4.0

REPOSITORY RECORD

Teodorescu, M., and Homer Rahnejat. 2019. "Newtonian Mechanics in Scale of Minutia". figshare. https://hdl.handle.net/2134/4683. 
This item was submitted to Loughborough's Institutional Repository (https://dspace.lboro.ac.uk/) by the author and is made available under the following Creative Commons Licence conditions.

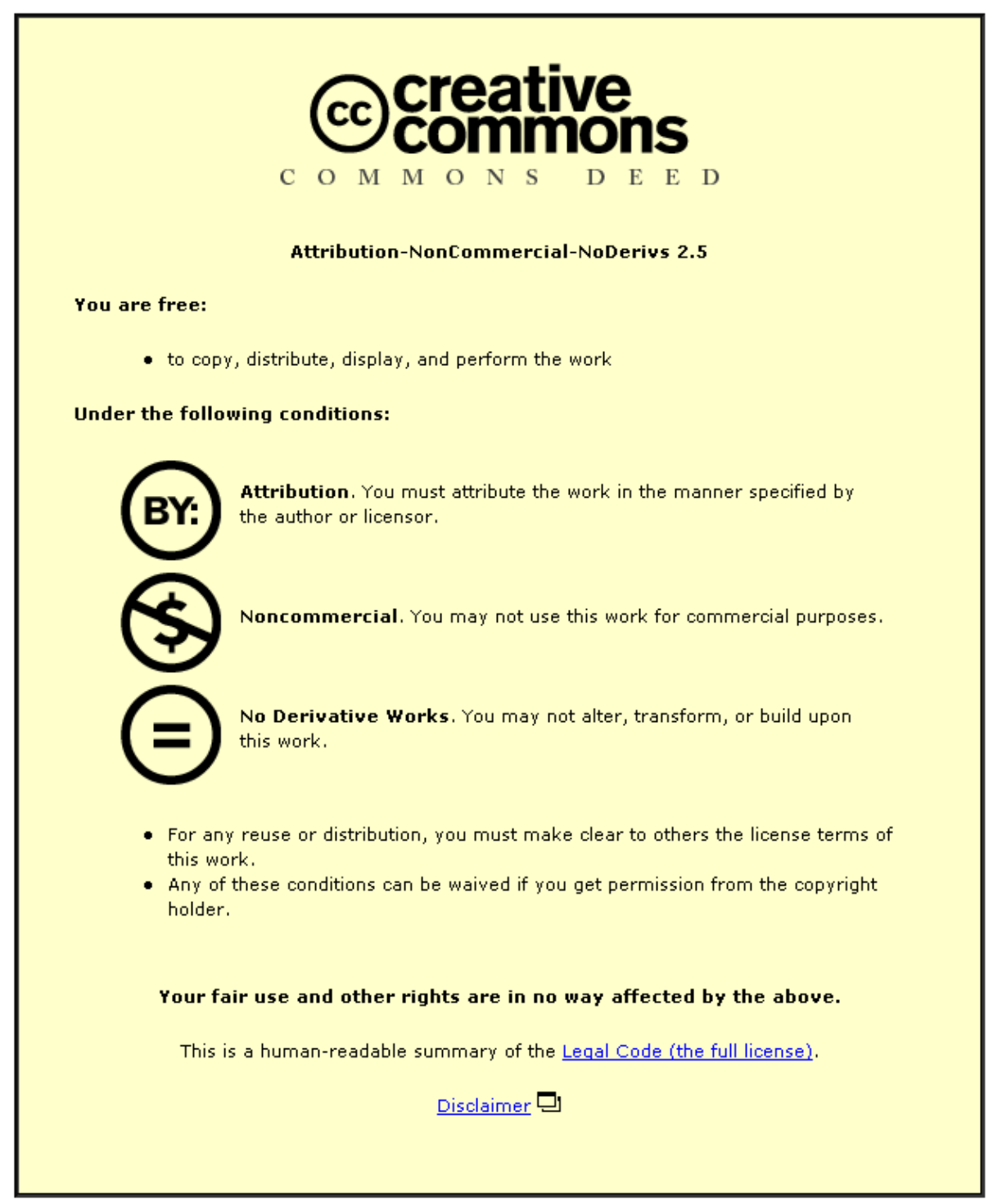

For the full text of this licence, please go to: http://creativecommons.org/licenses/by-nc-nd/2.5/ 


\title{
Newtonian mechanics in scale of minutia
}

\author{
M Teodorescu $^{1 *}$ and $\mathbf{H}$ Rahnejat ${ }^{2}$ \\ ${ }^{1}$ School of Engineering, Cranfield University, Cranfield, UK \\ ${ }^{2}$ Wolfson School of Mechanical and Manufacturing Engineering, Loughborough University, Loughborough, UK
}

The manuscript was received on 21 May 2008 and was accepted after revision for publication on 8 September 2008.

DOI: 10.1243/14644193JMBD163

\begin{abstract}
This article commemorates Newton's contributions to mechanics in small scale. In particular, it deals with Newtonian slow viscous action of fluids in narrow conjunctions leading to hydrodynamics. It is shown that the Newtonian continuum relies on some bulk properties of fluids as opposed to their molecular interactions. When the latter and surface energy effects become dominant, the interaction potentials deviate from the Newtonian continuum. A plethora of largely empirically based force laws are used to describe conjunctional behaviours in nanoscale, usually lightly loaded.

Some of these force laws are described, and their applicability to nanoconjunctions of very small devices and some biological systems is noted. In general, a thorough understanding of all the involved kinetics is required. Representative problems in soft nanoscale contacts in normal (humid) atmosphere are highlighted in the article. It is shown that contact load/adhesion depends on several key parameters including surface roughness, surface free energy, atmospheric moisture, and normal approach velocity.
\end{abstract}

Keywords: viscous flow, contact mechanics, micro- and nanoscale contacts, adhesion, van der Waals, meniscus, hydration

\section{INTRODUCTION}

Developments in the eighteenth century showed that the description of force in Newtonian mechanics was void of the nature of media or material of interacting bodies, except for their inertial properties (vis inertiae). As described by Rahnejat [1], Coulombic electrostatic force between opposite charges, while obeying the inverse distance squared force law in the same manner as the law of universal gravitation, is a function of the medium in which the charges reside [2]: $F=K q_{2} / r^{2}$ (i.e. $K$ is not a universal constant, whereas $G$ is), where $K=q_{1} / 4 \pi \varepsilon_{0} \varepsilon_{\mathrm{r}}$. The formulation of the electrostatic force by de Coulomb [2] was influenced by Newton's law of universal gravitation [3]. However, the inverse distance squared force law (as a central force) explicitly predicts an elliptical orbit, e.g. for any of the planets in the solar system, where the sun is

*Corresponding author: School of Engineering, Cranfield University, Cranfield, Bedfordshire MK43 OAL, UK. email: m.s.teodorescu@cranfield.ac.uk at one of the two foci. This is a non-uniform motion (i.e. accelerated motion: note the elliptical nature of the path and the centrifugal acceleration). Therefore, when de Coulomb laid the foundation for electrostatic force on the Newtonian law of universal gravitation, he probably did not realize the implicit acceptance of an elliptical path. If such charges undertake motion, e.g. in electrodynamics (motion of electrons around atomic nuclei), then according to Maxwell's electromagnetic theory [4], an electric charge in non-uniform motion would emit radiation (readers are referred to any standard text in electromagnetism), thus decreasing its own energy. Hence, all electrons would gradually fall towards the atomic nuclei, and Bohr's model of an atom cannot be upheld as realistic. To ensure that the atomic model remains realistic, Bohr had to resort to a non-classical theory [5-7] (other than Newtonian or relativistic), which would account for non-uniform motion of electrons as electrically charged particles and radiation of energy in discrete 'packets', which were then named as photons by Einstein [8]. This discretization of energy is known as quantization (one quanta being the photonic energy). Thus, a new theory 
was born and termed quantum mechanics. It is the mechanics, because it retained the particulate nature of moving charges such as electrons, with which quantization of their angular momenta is subject to an assortment of spin motions.

By the nineteenth century, it had become clear that the deviation from Newtonian kinetics is true of all forms of electromagnetic interactions and that in many cases the forces between particles and molecules do not in fact obey the inverse distance squared law. It also emerged that when these forces are very small compared with the gravitational force over large distances, they are considerably larger at short range. They can even be $\approx 10^{36}$ times greater than gravity in the molecular and atomic ranges, where gravity apparently plays no significant role. At the closest range, very significant binding forces occur such as the strong force at the atomic nuclei. It happens that the plethora of forces underlying various phenomena deviating from the Newtonian model fall into the scale of minutia (in the submicrometre and below) (Fig. 1). Furthermore, they need not be electromagnetic in nature, since many interactions governed by surface energy effects and topographical interactions also seemingly do not conform to Newtonian mechanics.

It became clear that retention of gravitational action potential in intermolecular range yields results that cannot be logically sustained. To show this, let the action potential be of the general form

$$
w(r)=-\frac{C}{r^{n^{\prime}}}
$$

Note that when $n^{\prime}=1$, the potential takes the form of gravitation with $C=G M$.

If a molecule of diameter $\varepsilon$ resides in a region of space (e.g. constrained between two solid boundaries), it would interact with all its neighbouring molecules. If one assumes the immediate neighbourhood of this molecule to be a spherical shell of radius $r$ and thickness $\mathrm{d} r$ and to contain other identical molecules with a number density $\rho$, then the total interaction energy for it is obtained by simple superposition of all the interactions as

$$
E=-\int_{\varepsilon}^{\ell} 4 \pi \rho r^{2} w(r) \mathrm{d} r
$$

where $\ell$ is the size of interaction domain. Now, substituting equation (1) into (2) yields

$$
E=-4 \pi \rho C \int_{\varepsilon}^{\ell} \frac{1}{r^{n-2}} \mathrm{~d} r=-\frac{4 \pi \rho C}{n^{\prime}-3}\left\{1-\left(\frac{\varepsilon}{\ell}\right)^{n^{\prime}-3}\right\}
$$

Note that when $\ell \gg \varepsilon$, the second term in the curly brackets should diminish as $\ell$ becomes larger (i.e. the interactions from distant molecules are less significant). If one retains $n^{\prime}=1$ (as in the gravitational action potential), in fact, the opposite would result as the ratio $\varepsilon / \ell \ll 1$. In fact, it is clear that $n^{\prime}>3$ would be required for a sensible interpretation to be made. This means that as the distance between a pair of molecules increases their kinetic interactions diminish rapidly, whereas with $n^{\prime}=1$, the gravitational attraction between large bodies remains significant over astronomical distances. Thus, for intermolecular actions, equation (3) becomes

$$
E=-\frac{4 \pi \rho C}{\left(n^{\prime}-3\right) \varepsilon^{n^{\prime}-3}}
$$

It transpires that for the various forms of kinetic interaction the value of $n^{\prime}$ differs, although the nature of intermolecular actions is invariably electrostatic. This result is still regarded as rather puzzling and is interpreted to be due to a lack of fundamental understanding of the nature of microcosm. Irrespective of seemingly compelling arguments based on observations, it seems illogical that $w \propto 1 / r^{n^{\prime}}, n^{\prime}=1$ in several picometre range and when greater than tens of nanometre, but the interactions in the intervening range follow action potentials, where $n^{\prime}>3$.

The deviation of force laws in microcosm from Newtonian mechanics is reminiscent of the philosophical quandary facing his pragmatic empirical approach in the seventeenth century highlighted in reference

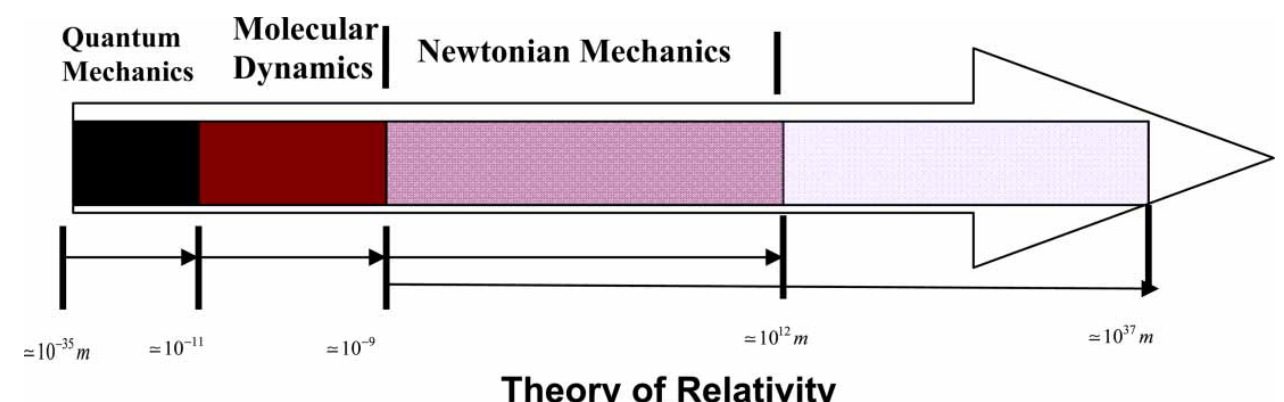

Fig. 1 Breadth of physics of motion 
[1] - one that was reconciled with a philosophical basis by him and his contemporaries. Thus, the overwhelming current view is that the rather empirical basis of some of the non-Newtonian force laws (theories fitting observations) in microcosm can be unified within a mechanical perspective. The basis upon which such unification is found diverges, depending on the philosophical perspective: one advocating determinism of interaction characteristics (a positivist approach) and another of stochastic nature. These competing arguments are within atomism itself.

Figure 1 shows the breadth of the 'understood' physics of motion. This is from $10^{-44}$ being the Planck time [9]; the time taken for a photon of light to traverse the Planck length (shortest conceived measure) $l=\left(\hbar G / c^{3}\right)^{1 / 2} \approx 10^{-35} \mathrm{~m}$, thus $\tau_{\mathrm{p}}=l / c \approx 10^{-44} \mathrm{~s}$ to the edge of the perceived universe, being 14 billion light years $\left(\approx 10^{29} \mathrm{~s}\right)$, a distance of $\left(\approx 10^{37} \mathrm{~m}\right)$. It happens that the law of universal gravitation is proven to hold at least within the solar system, extending to the Oort cloud, a distance of $7.5 \times 10^{12} \mathrm{~m}$ (and most likely well beyond). The only marginal error is noted for perihelion drift in motion of Mercury by $1^{\circ}$, a cumulative error over 8000 Mercury years calculated by Einstein [10] Taking this (modestly) to be the upper bound of Newtonian mechanics and the viscous action of molecules in conjunctions of tens of nanometre to be its lower bound is indicative of the great position in which he is held. The key point is that Newtonian mechanics is not confined to the scheme of large scale or that of the law of universal gravitation.

\section{NEWTONIAN MECHANICS IN SCALE OF MINUTIA}

This article concentrates on the scale of minutia related to the lower bounds of Newtonian physics. In particular, practical examples in micro-electromechanical systems and some in nature itself (such as that typical of tiny conjunctions of sticky feet of geckos and insects) are considered. Newtonian viscous flow action represents the underpinning of the fluidic conjunctions in which layers of assumed spherical undeformable molecules undertake relative motion in shear. Resistance to relative motion gives rise to fluid viscosity, as defined by Newton. Defining a unit element of fluid flow (containing many such molecules), Navier [11] and Stokes [12] formulated the Newtonian viscous flow model. The Navier-Stokes equations are the cornerstone of fluid flow problems. They take into account the inertial, gravitational (body), viscous, and surface forces, whose net effect gives rise to the induced flow. Unlike the upper bound of Newtonian mechanics, where the universal gravitational constant is independent of substance/physical properties of matter, at its lower bound, the Newtonian mechanics takes into account such attributes (e.g. viscosity and density), but not all (e.g. temperature). In fact, Stokes' unit element of flow necessarily contains many molecules of fluid, thus the physical parameters associated with the viscous flow model have bulk characteristics, as opposed to molecular, which are largely electromagnetic. Therefore, it is not surprising that any deviation from a continuum would breach Newtonian mechanics. This deviation would need to abrogate the principle of flow continuity in the same manner that quantization of energy defies relativistic interpretation in quantum mechanics. Ironically, Newtonian atomistic view at large scale (action-at-adistance) does not extend to the microscale, where assumption of a continuum forms the basis of interpretation. In fact, Navier's initial attempt was to introduce internal friction at the molecular level. It now transpires that his initial attempt could have extended Newtonian mechanics, had internal friction was formulated in terms of electromagnetic behaviour of molecules, a concept which was not understood for another quarter century (de Coulomb [2] and Maxwell [4]).

\subsection{Generalized Newtonian fluid flow}

In Principia [3], Newton devoted his attention to the flow properties of fluids in terms of their molecular action. He described the viscosity of a fluid as a measure of its resistance to flow, and defined the flow characteristics in terms of the effect of their viscosity and shear stresses upon the shear strain rate for the liquids that have become known as Newtonian fluids. His model for the fluid flow was based upon layers of molecules, as tiny spheres, rolling along between solid boundaries. As the liquids wet and cling to the adjacent solid surfaces, those next to a stationary boundary stagnate, whereas those in contact with a moving boundary follow it in concert. The layers between stationary and moving surfaces would move according to a linear velocity distribution through the thickness of the fluid film, directly proportional to the distance from the two solid boundaries. This type of distribution moving in orderly parallel layers is a stream-line flow. Since the relative motion of layers occurs against the resistance of the fluid, it is referred as viscous flow. Newton defined the interfacial shear strain rate between these layers of fluid as

$$
\frac{\mathrm{d} \gamma}{\mathrm{d} t}=\frac{1}{h_{i}} \frac{\mathrm{d} s_{i}}{\mathrm{~d} t}=h_{i}^{-1} \dot{s}_{i} \in i
$$

Newton further showed that the friction force required at the moving surface to maintain its velocity, $u$, must be proportional to the wetted area of contact and the velocity gradient (in other words, the shear strain rate). 
Thus

$$
F=\eta A h^{-1} \dot{s}
$$

where $h$ is the thickness of the fluid channel, $\dot{s}=\dot{s}_{i}$ $(i=1)$ is the sliding velocity of the moving solid surface, and $\eta$ is the constant of proportionality, defined by Newton as the absolute viscosity of the fluid or the dynamic viscosity as it is referred to today. It is defined as

$$
\eta=\frac{\tau}{\dot{\gamma}}
$$

Note that this is a bulk property of the fluid belonging to an assumed continuum.

Navier [11] and Stokes [12] are attributed with the derivation of equations of motion for Newtonian fluids. In fact, their derivations were quite different. Pointedly, Navier attempted to modify the Newtonian model to include what he termed as the molecular interaction force. On the other hand, Poisson [13], having dismissed Navier's internal friction force, derived the Newtonian viscous flow model by considering the equilibrium of an element of fluid, an approach due to Euler. Although Poisson's solution was 2 years before that of Stokes [12], for some unknown reason, the priority for the inclusion of a surface force due to shear stress has been given to Stokes. The shear stress tensor can be represented as

$$
\tau^{i j}=\eta\left(\frac{\partial \boldsymbol{v}^{i}}{\partial \boldsymbol{x}^{j}}+\frac{\partial \boldsymbol{v}^{j}}{\partial \boldsymbol{x}^{i}}\right)
$$

where $\boldsymbol{v}^{i}$ is the velocity vector along the coordinate vector $\boldsymbol{x}^{i}$. The differential terms in equation (8) indicate the distortion of an element of fluid. Clearly, for state of equilibrium: $\boldsymbol{\tau}^{i j}=\boldsymbol{\tau}^{j i}$ for $i, j=1-3$, where 1,2 , and 3 represent the spatial coordinates (e.g. $x, y$, and $z)$. The hydrostatic pressure $p_{\mathrm{H}}$ in the fluid is considered to be the average of the three normal stress tensor components, as

$$
p_{\mathrm{H}}=-\frac{1}{3}\left(\sum_{i}\left\|\sigma^{i}\right\|\right)
$$

Now, the stress tensor for direct components is given as

$$
\sigma^{i}=-p_{\mathrm{H}}+v \nabla \boldsymbol{v}^{i}+2 \eta \boldsymbol{v}_{, i}^{i}
$$

Note that the term $\nabla \boldsymbol{v}^{i}$ is the divergence of the velocity vector $\boldsymbol{v}^{i}$ and $v$ the secondary viscosity coefficient, and also that the physical meaning of divergence of the velocity vector is the rate in which the fluid flows out of a given point. This is sometimes referred as dilatation. Now letting $i=1-3$ in equation (10) and replacing in equation (9), one arrives at the condition $(3 v+2 \eta) \nabla \boldsymbol{v}^{i}=0$, which yields $\boldsymbol{v}=-2 \eta / 3$. The surface normal and the tangential viscous forces that move the element of fluid in the coordinate directions $\boldsymbol{x}^{i}$ are given as $\boldsymbol{N}^{i}=\sigma_{, i}^{i} \mathrm{~d} V$ and $\boldsymbol{T}^{i}=\tau_{, j}^{i j} \mathrm{~d} V$, where $\mathrm{d} V=\sum_{i} \mathrm{~d} \boldsymbol{x}^{i}, i=1-3$.

The external forces or a gravitational force applied to the bulk of the fluid continuum move the element of fluid in an accelerated manner. Denoting these forces per unit mass of the fluid continuum as $\boldsymbol{X}^{i}$, the body forces per element of fluid are given as $\rho \boldsymbol{X}^{\mathrm{i}} \mathrm{d} V$. Note that the force vector $\boldsymbol{X}^{i}$ is in the same form as the generic Eulerian equation of motion and, therefore, has the units of acceleration. Thus, as in the case of Euler's equation, $\rho$ can be regarded as the force density of the element in the continuum and has the appropriate units of $\mathrm{Ns}^{2} / \mathrm{m}^{4}$.

The velocity vector of an element of fluid can be represented in space-time as $\boldsymbol{v}^{i}=f\left(\boldsymbol{x}^{i}\right), i=0-3$, where $x^{0}=t$ denotes the temporal coordinate. Therefore, the change in the velocity vector can be obtained with respect to the coordinate system $\boldsymbol{x}^{i}$ as $\delta \boldsymbol{v}_{i}=$ $\left(\partial \boldsymbol{v}^{i} / \partial \boldsymbol{x}^{i}\right) \delta \boldsymbol{x}_{i}$, where Einstein's summation convention applies over the repeated index $i$. The equation can be rewritten as $\delta \boldsymbol{v}_{i} / \delta \boldsymbol{x}_{0}=\left(\partial \boldsymbol{v}^{i} / \partial \boldsymbol{x}^{i}\right)\left(\delta x_{i} / \delta x_{0}\right)$, and using the properties of limits in differential geometry as $\delta x_{0} \rightarrow 0$ : $\delta x_{i} / \delta x_{0}=\mathrm{d} x^{i} / \mathrm{d} t=\boldsymbol{v}^{i}$, and: $\delta \boldsymbol{v}_{i} / \delta \boldsymbol{x}_{0}=\mathrm{d} \boldsymbol{v}^{i} / \mathrm{d} t$. Thus

$$
\begin{aligned}
\frac{\mathrm{d} \boldsymbol{v}^{i}}{\mathrm{~d} t} & =\frac{\partial \boldsymbol{v}^{i}}{\partial t}+\boldsymbol{v}^{i} \frac{\partial \boldsymbol{v}^{i}}{\partial x^{i}}+\boldsymbol{v}^{j} \frac{\partial \boldsymbol{v}^{i}}{\partial x^{j}}, \quad i=1-3, \\
j & =1-3, \quad i \neq j
\end{aligned}
$$

Note that the term on the left-hand side of this equation provides the total derivative of the velocity vector $\boldsymbol{v}^{i}$ (i.e. with respect to both time and spatial coordinates) and is referred as the Stokesian derivative. The results are three equations for $i=1-3$. The first term on the right-hand side gives the variation of the velocity vector at a given position in space-time and is often termed as the local derivative. The other terms on the right-hand side are the connective terms, providing the spatial characteristics of the variation of the velocity vector as measured in a chosen coordinate system. The inertial forces necessary to accelerate the elemental fluid volume $\mathrm{d} V$ are obtained as $\boldsymbol{F}^{i}=$ $\rho(\mathrm{d} t / v) \mathrm{d} V, i=1-3$. Imposing Eulerian dynamic equilibrium for the elemental volume $\mathrm{d} V$ for the above stated forces yields

$$
\begin{aligned}
& \boldsymbol{F}^{i}=\rho \frac{\mathrm{d} \boldsymbol{v}^{i}}{\mathrm{~d} t}=\rho \boldsymbol{X}^{i}+\boldsymbol{N}^{i}+\boldsymbol{T}^{i j} \\
& =\rho \boldsymbol{X}^{i}+\sigma_{, i}^{i}+\tau_{, j}^{i j}, \quad \text { or simply } \\
& \rho \frac{\mathrm{d} \boldsymbol{v}^{i}}{\mathrm{~d} t}=\rho \boldsymbol{X}^{i}+\sigma_{, i}^{i}+\tau_{, j}^{i j}
\end{aligned}
$$

This is merely the reinstatement of Newton's second axiom and Newton-Euler formulation with an additional term, being the last term on the right-hand 
side of equation (11). This was the additional term that Navier was attempting to add to the Euler's equation the term that was defined finally correctly by SaintVenant. This equation can be represented in terms of pressure $p$. The resulting equations of motion are known as the Navier-Stokes equations

$$
\begin{aligned}
\rho \frac{\mathrm{d} \boldsymbol{v}^{i}}{\mathrm{~d} t}= & \rho \boldsymbol{X}^{i}-\frac{\partial p}{\partial \boldsymbol{x}^{i}}-\frac{2}{3} \frac{\partial}{\partial \boldsymbol{x}^{i}}\left(\eta \nabla \boldsymbol{v}^{i}\right)+2 \frac{\partial}{\partial \boldsymbol{x}^{i}}\left(\eta \frac{\partial \boldsymbol{v}^{i}}{\partial \boldsymbol{x}^{i}}\right) \\
& +\frac{\partial}{\partial \boldsymbol{x}^{j}}\left[\eta\left(\frac{\partial \boldsymbol{v}^{i}}{\partial \boldsymbol{x}^{j}}+\frac{\partial \boldsymbol{v}^{j}}{\partial \boldsymbol{x}^{i}}\right)\right] \\
= & \rho \boldsymbol{X}^{i}-p_{, i}-\frac{2}{3}\left(\eta \nabla \boldsymbol{v}^{i}\right)_{, i}+2\left(\eta \boldsymbol{v}_{, i}^{i}\right)_{, i}+\left[\eta\left(\boldsymbol{v}_{, j}^{i}+\boldsymbol{v}_{, i}^{j}\right)\right]_{, j}
\end{aligned}
$$

with the Stokesian derivative $\mathrm{d} \boldsymbol{v}^{i} / \mathrm{d} t=\boldsymbol{v}_{, 0}^{i}$. Then, the three Navier-Stokes equations in their most general form are

$$
\rho \boldsymbol{v}_{, 0}^{i}=\rho \boldsymbol{X}^{i}-p_{, i}-\frac{2}{3}\left(\eta \nabla \boldsymbol{v}^{i}\right)_{, i}+2\left(\eta \boldsymbol{v}_{, i}^{i}\right)_{, i}+\left[\eta\left(\boldsymbol{v}_{, j}^{i}+\boldsymbol{v}_{, i}^{j}\right)\right]_{, j}
$$

Note that for each of the equations $i=1-3, j=1-3$, $i \neq j$, the summation on the right-hand side of the equation takes place over the repeated indices $i$ and $j$.

For an elemental volume $\mathrm{d} V$, the mass flow per unit measures of time and area (referred as the mass flux) through a surface is given by the product of the normal velocity vector to that surface and the density as $\rho \boldsymbol{v}^{i}$. The mass flux changes from position to position in the elemental volume. Therefore, one can assume it to be $\rho v^{i}$ at the centre of the element with the net outflow as

$$
\begin{aligned}
& \text { outflow }=\left(\rho \boldsymbol{v}^{i}+\frac{1}{2}\left(\rho \boldsymbol{v}^{i}\right)_{, i} \mathrm{~d} x^{i}\right) \mathrm{d} x^{j} \mathrm{~d} x^{k} \\
&-\left(\rho \boldsymbol{v}^{i}-\frac{1}{2}\left(\rho \boldsymbol{v}^{i}\right)_{, i} \mathrm{~d} x^{i}\right) \mathrm{d} x^{j} \mathrm{~d} x^{k}, \\
& i=1-3, \quad j=1-3, \quad k=1-3, \quad i \neq j \neq k
\end{aligned}
$$

The rate of decrease in mass in the elemental volume $\mathrm{d} V$ is $-\left(\partial \rho / \partial x^{0}\right) \mathrm{d} V, x^{0}=t$, which can be represented as

$$
\rho_{, 0} \mathrm{~d} V=\rho_{, 0} \mathrm{~d} x^{i} \mathrm{~d} x^{j} \mathrm{~d} x^{k}
$$

Equating equations (14) and (15) leads to the Stokes' continuity of flow equation

$$
\rho_{, 0}+\left(\rho \boldsymbol{v}^{i}\right)_{, i}=0
$$

The pair of equations (13) and (16), each of which provides three equations, is sufficient to evaluate the unknowns $p, \boldsymbol{v}^{i}, i=1-3$, provided fluid viscosity and density can be described in their terms. Of course, such relationships exist.

\section{REYNOLDS AND NEWTONIAN SLOW VISCOUS FLOW MODEL: SCALE OF MINUTIA}

The class of interest here (i.e. fluid flow in the scale of minutia) is known as the slow viscous motion, where the pressure and viscous terms become dominant and one can safely neglect the body and inertial forces. This class of problem is generally regarded as hydrodynamics, usually in tribological conjunctions. The dilatation term (i.e. the divergence of the velocity vector) can also be ignored because of the incompressible nature of most lubricants. Therefore, the Navier-Stokes equations simplify to $p_{, i}=\eta\left[\left(\boldsymbol{v}_{, i}^{i}\right)_{, i}+\left(\boldsymbol{v}_{, j}^{i}\right)_{j}+\left(\boldsymbol{v}_{, k}^{i}\right)_{, k}\right]$. The term in the square bracket is the second derivative of the velocity vector with respect to the spatial coordinates as defined by Laplace. This term is referred as Laplacian and is denoted by $\nabla^{2} \boldsymbol{v}^{i}=\boldsymbol{v}_{l m}^{i}$, where $l m$ means subsequent differentiations with respect to the indices $l$ and $m$, where $l=i, j, k, m=i, j, k$. Hence, the simplified Navier-Stokes equation for incompressible slow viscous motion becomes

$$
p_{, i}=\eta \nabla^{2} \boldsymbol{v}_{, l m}^{i}
$$

Reynolds observed that the pressure gradient across a film of lubricant could be ignored due to its thinness. Also, order of magnitude analysis shows that variation of velocity vector components with respect to the same coordinate (i.e. $z$ ) is large compared with all other velocity gradients. This is due to the geometry of any fluid film (the dimensions of a very small conjunction are insignificant compared with the size of its solid barriers). Therefore, $\boldsymbol{v}_{l m}^{i}=$ 0 , where for $i=1,2, l=m=1,2$ and for $i=3, l=$ $m=1,2,3$ (in other words $\partial^{2} \boldsymbol{v}^{i} / \partial x^{2}=\partial^{2} \boldsymbol{v}^{i} / \partial y^{2}, i=1,2$, and $\left.\partial^{2} \boldsymbol{v}^{i} / \partial x^{2}=\partial^{2} \boldsymbol{v}^{i} / \partial y^{2}=\partial^{2} \boldsymbol{v}^{i} / \partial z^{2}, i=3\right)$. Denoting the position of contiguous surfaces along the $x^{3} \equiv z$ axis and with no slip at the boundaries, these idealized conditions further simplify the Navier-Stokes equations for isothermal assumption (no viscosity variation across the film) to $p_{, i}=\eta \boldsymbol{v}_{k k}^{i}$. Integrating twice with respect to the index $k$ and with no slip boundaries of the Newtonian viscous flow model, the boundary conditions yield

$$
\boldsymbol{v}^{i}=-z\left(\frac{h-z}{2 \eta}\right) p_{, i}+\frac{1}{h}\left[(h-z)\left\|\boldsymbol{v}_{1}^{i}\right\|+z\left\|\boldsymbol{v}_{0}^{i}\right\|\right]
$$

Using the viscous shear stress tensor and noting that the first term in the bracket is much smaller than the second, $\tau^{k i}=\eta \boldsymbol{v}_{, k}^{i}$. Substituting from equation (8), the interfacial viscous shear stresses acting on the 
adjacent solid barriers are

$$
\begin{aligned}
& \tau_{0}^{k i}=\eta \boldsymbol{v}_{, k}^{i}=-\frac{h}{2} p_{, i}-\frac{\eta}{h}\left\|\boldsymbol{v}_{1}^{i}-\boldsymbol{v}_{0}^{i}\right\| \\
& -\tau_{h}^{k i}=\eta \boldsymbol{v}_{, k}^{i}=-\frac{h}{2} p_{, i}+\frac{\eta}{h}\left\|\boldsymbol{v}_{1}^{i}-\boldsymbol{v}_{0}^{i}\right\| \\
& \text { where } i=1,2 \quad \text { and } \quad k=3
\end{aligned}
$$

If one denotes the volume flow rates per unit width in the spatial directions: $x^{i}, i=1,2$ as $\boldsymbol{q}_{, i}$, then $\boldsymbol{q}_{, i}=$ $\int_{x^{k}} \boldsymbol{v}^{i} \mathrm{~d} x^{k}=\int_{0}^{h} \boldsymbol{v}^{i} \mathrm{~d} x^{k}$. One can replace for the velocity vector from equation (18), thus $\left\|\boldsymbol{q}_{, i}\right\|=-\left(h^{3} / 12 \eta\right) p_{, i}+$ $h / 2\left\|\boldsymbol{v}_{0}^{i}+\boldsymbol{v}_{1}^{i}\right\|$, for all $i=1,2$.

Now, the continuity of flow equation (16) can be made use of by replacing in it the velocity vector components from equation (18). It is mathematically convenient to represent the continuity equation in an integral form. This yields

$$
\begin{gathered}
\int_{0}^{h}\left[\rho_{, 0}+\left(\rho \boldsymbol{v}^{i}\right)_{, i}\right] \mathrm{d} x^{k}=\int_{0}^{h} \rho_{, 0} \mathrm{~d} x^{k}+\int_{0}^{h}\left(\rho \boldsymbol{v}^{i}\right)_{, i} \mathrm{~d} x^{k} \\
\quad=h \rho_{, 0}-\left.\rho \boldsymbol{v}^{i}\right|^{z=h} h_{, i}+\left(\int_{0}^{h} \rho \boldsymbol{v}^{i} \mathrm{~d} x^{k}\right)_{, i} \\
\quad=h \rho_{, 0}-\rho\left\|\boldsymbol{v}_{1}^{i}\right\| h_{, i}+\left(\int_{0}^{h} \rho \boldsymbol{v}^{i} \mathrm{~d} x^{k}\right)_{, i}
\end{gathered}
$$

where $i=1-3, k=3$. When $i=k=3$, $\left(\int_{0}^{h} \rho \boldsymbol{v}^{i} \mathrm{~d} x^{k}\right)_{, i}=$ $\left(\int_{0}^{h} \rho \boldsymbol{v}^{k} \mathrm{~d} x^{k}\right)_{, k}=\rho\left\|\boldsymbol{v}^{k}\right\|$. Then, the integrated continuity equation becomes

$$
h \rho_{, 0}-\rho\left\|\boldsymbol{v}_{1}^{i}\right\| h_{, i}+\left(\rho \int_{0}^{h} \boldsymbol{v}^{i} \mathrm{~d} x^{k}\right)_{, i}+\rho\left\|\boldsymbol{v}^{k}\right\|=0
$$

The integral term in equation (16) for $i=1,2$ is given by volume flow rates in the $x$ and $y$ directions. Hence $\left(\rho\left\|\boldsymbol{q}_{i}\right\|\right)_{, i}-\rho\left\|\boldsymbol{v}_{1}^{i}\right\| h_{, i}+\rho\left\|\boldsymbol{v}^{k}\right\|+h \rho_{, 0}=0$. This is the Reynolds' equation, which upon replacement for the volume flow rates and re-arrangement of terms for a constant force density $\left(\rho_{0}=0\right)$ can be represented in its familiar form as

$$
\begin{aligned}
\left(\frac{\rho h^{3}}{12 \eta} p_{, i}\right)_{, i}= & \left(\frac{\rho h}{2}\left\|\boldsymbol{v}_{0}^{i}+\boldsymbol{v}_{1}^{i}\right\|\right)_{, i} \\
& -\rho\left\|\boldsymbol{v}_{1}^{i}\right\| h_{, i}+\rho\left\|\boldsymbol{v}_{1}^{k}-\boldsymbol{v}_{0}^{k}\right\|
\end{aligned}
$$

From Reynolds [14] to date, this equation has been used for the description of fluid flow in conjunctions of the order of micrometre. However, thin hydrodynamic films have very low load-carrying capacity given by the integrated pressure distribution

$$
W=\int_{i} \int_{j} p_{i j} \mathrm{~d} x^{i} \mathrm{~d} x^{j}
$$

Consequently, breaches of lubricant film were expected, and thus the absence of wear scars was puzzling to Reynolds. Unknown to him, localized deformation of elastic solids in contact had been formulated by Hertz [15], which after a considerable period of time led to the topic of elastohydrodynamics (the historical discourse is beyond the scope of this article, readers are referred to any standard text on the subject). The key point is that localized deformation of contiguous solids under load results in an enhanced gap, where a film of fluid can reside at high pressure.

\section{NEWTON CALCULUS OF VARIATION AND CLASSICAL HERTZIAN THEORY}

In Principia [3], Newton devoted much work to the calculus of variations with evanescent quantities. These were covered by lemmas, a number that deal with properties of differential geometry in the limits. It is then clear that for a tangent to a solid of revolution the angle of contact should vanish for the curve to continue beyond it (Lemmas VI and VII). This means that for infinitesimal localized deformation of solids of revolution, where $\delta \ll R$, the contact footprint covers an infinitesimal area around the tangent point to their curvature. Using the chordal theory (Fig. 2), $x^{2}=(2 R-z) z$.

This yields $z=x^{2} / 2 R, R \gg z$, when: $x \rightarrow 0$ (tangent), $\mathrm{d} z / \mathrm{d} x \rightarrow 0$ and: $\mathrm{d}^{2} z / \mathrm{d} x^{2} \rightarrow 1 / R$ (curvature). Thus, a parabolic shape may be assumed in very small regions around the tangent point. Hertz [15] used these Newtonian lemmas to undertake small plane-strain localized deformation of solids of revolution in contact and under load. Then, two points on the surfaces of two such rollers, with radii $r_{1}$ and $r_{2}$, initially touching and then loaded against each other by a load

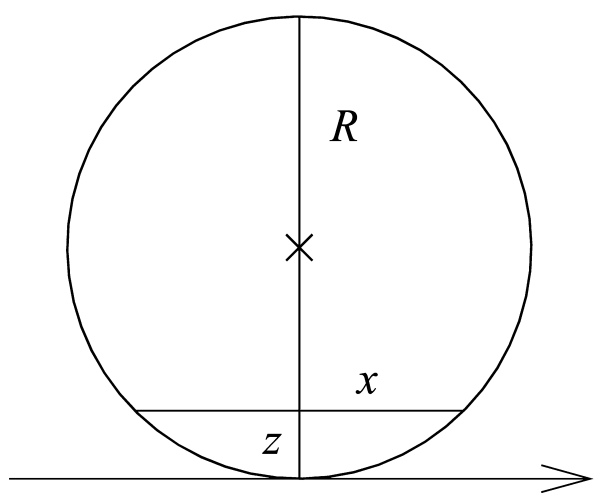

Fig. 2 Geometry of a localized contact 
$W$ deform along their contact normal by amounts $\delta_{0 i}, i=1,2$. At the coordinate location $x$ within the contact, the deflection is $\delta=\sum_{i} \delta_{0 i}-x^{2} \sum_{i} 1 / 2 r_{i}$. Now, if the contact semi-half-width is given as $a$, then $1 / a(\delta-$ $\left.\sum_{i} \delta_{0 i}\right)=a^{2} / 2 a\left(\left(1 / r_{1}\right)+\left(1 / r_{2}\right)\right)$ and letting $D=(\delta-$ $\left.\sum_{i} \delta_{0 i}\right)$, then $D=a / 2\left(\left(1 / r_{1}\right)+\left(1 / r_{2}\right)\right)$.

By elementary elasticity, $D / a \propto\left(p_{\mathrm{m}} / E^{\prime}\right)=a / 2\left(\left(1 / r_{1}\right)\right.$ $\left.+\left(1 / r_{2}\right)\right)$; thus, $p_{\mathrm{m}} \propto\left(a E^{\prime} / R_{\mathrm{e}}\right)$. Now, the mean pressure for two rollers of length $L$ in contact is $p_{\mathrm{m}}=W / 2 a L$. The last two relations result in $W=k\left(L E^{\prime} / R_{\mathrm{e}}\right) a^{n}$. Simple dimensional analysis shows that $n=2$. Also, for an equivalent roller of radius $R$ against a semi-infinite solid, $a=\sqrt{R_{\mathrm{e}} \delta}$, which leads to the Hertzian contact load of $W=k L E^{\prime} \delta=K \delta$. This equation, however, does not take into account the pressure rises (spikes) at the edges of a roller penetrating into a flat [16]. Correcting these, a more complex relationship results

$$
W=\frac{\pi L E^{\prime}}{2\left\{\ln \frac{2 L}{a}+\frac{1}{2}\right\}} \delta \approx K \delta
$$

Using Newton-Euler approach, the localized Hertzian impact of a roller on a semi-infinite elastic half-space is given as

$$
m \ddot{\delta}=-W
$$

Replacing from equation (24) and by integration

$$
\frac{1}{2}\left(\dot{\delta}^{2}-v^{2}\right)=-\frac{K}{2 m} \delta^{2}, \quad \text { or simply } \quad \dot{\delta}^{2}=v^{2}-\frac{K}{2 m} \delta^{2}
$$

Newton and Poisson did not consider the localized deformation of impacting solids, thus the impact time was considered to be zero. Equation (26) represents the principle of conservation of energy, referred by Leibniz [17], as vis viva (the living force). Newton preferred the principle of conservation of momentum, which only holds unconditionally for rigid impact of bodies, or $\dot{\delta}^{2}=v^{2}$, thus: $\delta=v$.

If one considers a wet impact, then $W$ is the integrated pressure distribution resulting from equation (23) and $\delta_{i j}=\left(1 / E^{\prime}\right) I_{i j}^{k l} p_{k l} \mathrm{~d} x^{l} \mathrm{~d} x^{k}$ not the dry impact resulting from the solution of equation (24), where $\ddot{\delta}$ is replaced by $\ddot{z}$ (note that $\delta \neq z$ is clear from the instantaneous gap given as (Fig. 3) $h=h_{0}+x^{2} / 2 R+\delta$. Such a solution represents an elastohydrodynamic impact $[18,19]$.

\section{NANOSCALE CONJUNCTIONS - MOLECULAR INTERACTIONS AND SURFACE ENERGY EFFECTS}

The above discussions illustrate the profound influence of Newton on subsequent discoveries as well as hitherto, the broadest range of applications in

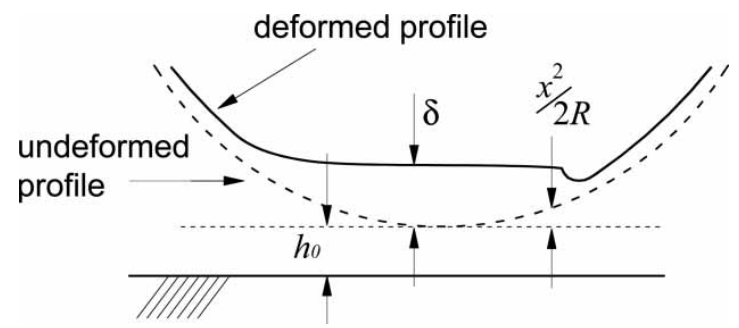

Fig. 3 An elastohydrodynamic conjunction

physics of motion. Newtonian physics is applied in the scale of minutia, where a continuum is assumed and the principle of causality is adhered. With a growing trend in system down-sizing, molecular behaviour in submicron and nanoscales has become prevalent. Observations have shown deviant behaviour of matter from that expected in a continuum. These have given rise to analyses that are commonly referred as non-Newtonian such as thixotropy of thin fluid films at high shear rates [20] and density variations near ultra-smooth constraining solid barriers [21-24]. As shown in section 1, material points in such environments do not comply with the Newtonian potential. Empirical formulations or statistical approaches have been the common thread for all such deviant behaviour. There is per se no fundamental objection to the empirical nature of force laws in the scale of minutia. After all, the law of universal gravitation was also empirical (based on a model fitting the observations of Kepler [25]). How insignificant its effect may be at close range as shown in reference $[\mathbf{1}]$, the profound nature of that law is its simplicity and universality. The problem with other observationbased force laws is their limited range, their complexity, and the widely felt intuition that nature is unlikely to act with such a plethora of interactions. To illustrate the problem in small scale, the article presents conjunctions that are subjected to a combination of such forces. These contacts are typified by light loads, surface interactions, and intermolecular behaviour. They are prevalent in micromechanisms and biological systems such as sticky feet of insects and geckos. Depending on prevailing conditions, dominant forces may include adhesion, van der Waals interactions, and hydration among others [26-30].

Under normal atmospheric conditions, water molecules condense on surfaces according to the level of atmospheric humidity. The condensation process depends on a threshold free energy level that determines the condensation time and the height of menisci bridges, which form between the asperities of any pair of contiguous surfaces [31]. Therefore, partial wetness of the contact is governed by level of humidity and condensation activation time. The wet portion of the contact is subjected to a balance of 
a number of forces; meniscus action, van der Waals forces, and at closer range hydration. The hydration force has a repulsive nature and is one of the least understood forces. It has been suggested that it is merely a form of solvation in water with a purely oscillatory behaviour [32], whereas others have proposed a monotonic exponential repulsion or attraction [33]. However, observations show that the hydration force is overall repulsive with an oscillatory behaviour below a gap of $4 \mathrm{~nm}[\mathbf{3 4}, \mathbf{3 5}]$. The oscillatory behaviour is attributed to the molecular packing order of water molecules near a fairly smooth surface, whereas there have been various explanations for the very short range monotonic exponential behaviour acting above the diminishing DLVO (Derjaguin and Landau [36], and Verwey and Overbeek [37]) contribution in such a range. This can be attributed to the hydrophobic interaction that can rupture or resist water menisci near the hydrophobic surfaces. For example, hydration plays an important role in the tribology of skin, with $\beta$-carotene being hydrophobic.

Due to the hierarchical structure of the rough surfaces, each element of the topology encounters roughness levels proportional to its size. The present analysis is confined to nanoscale irregularities of the contact. It is assumed that the contact between the asperity tips is smooth on a subnanometre scale.

Between completely dry surfaces, only van derWaals interaction is thought to act, whereas between wet surfaces meniscus bridges would form and additional forces can play a role. It is very important to determine the number of meniscus bridges formed between the two contiguous rough surfaces. Currently, there are two accepted mechanisms for nanobridge formation between surfaces. One presumes pre-existence of a thin layer of water on the surface $[\mathbf{3 8}, \mathbf{3 9}]$. Then, the number of formed bridges can be computed [38]. The other assumes that the surfaces are dry and if they remain in close proximity for a sufficiently long period, a necessary quantity of water would condense upon them for at least a monolayer of water to form. This sufficient period of time is referred as the activation time, prior to which no meniscus bridge would be formed. In post monolayer formation, the depth of water grows following a logarithmic law $[\mathbf{3 1}, \mathbf{4 0}]$. In reality, both described mechanisms of menisci formation would probably contribute.

The current work considers the contribution of several interacting mechanisms. However, to compute the total force acting on a surface, those applied to an individual asperity tip should first be considered. Asperities are considered to be normally distributed, where the Gaussian height can be expressed as

$$
\phi(z)=\frac{1}{\sigma_{\mathrm{p}} \sqrt{2 \pi}} \mathrm{e}^{-z^{2} / 2 \sigma_{\mathrm{p}}^{2}}
$$

Between any arbitrary limits $z_{1}$ and $z_{2}$, the number of participating asperities is computed as

$$
n=S \frac{N}{\sigma_{\mathrm{p}} \sqrt{2 \pi}} \int_{z_{1}}^{z_{2}} \mathrm{e}^{-z^{2} / 2 \sigma_{\mathrm{p}}^{2}} \mathrm{~d} z
$$

The contribution of these asperities can be in one of three forms. These include contributions of dry asperities with van der Waals interactions only, whereas wet ones would induce meniscus action in addition to reduced van der Waals interaction through water as well as hydration at a closer range. A third group of asperities are those that have been wetted and become completely submerged; their contribution being through hydration only.

Electrostatic effect may be neglected as it is small, unless there is significant salt concentration in water. Therefore, at any approach of contiguous surfaces, the asperity interactions would be an amalgam of these three states.

For an example, the equivalent root mean square roughness of the contiguous surfaces is $\sigma_{\mathrm{p}}=0.5 \mathrm{~nm}$.

\section{1 van der Waals attraction}

As long as the surfaces are completely dry, van der Waals forces alone would account for their interaction. For a pair of hemispherical asperities with a gap of $D-z$ (Fig. 4), the van der Waals force per asperity interaction can be obtained as [41]

$$
f_{\mathrm{W}}=-\frac{A_{\mathrm{H}}}{6(D-z)^{2}} R_{\mathrm{e}}
$$

where $R_{\mathrm{e}}=\left(\left(1 / r_{\mathrm{asp} 1}\right)+\left(1 / r_{\mathrm{asp} 2}\right)\right)^{-1}$ is the equivalent radius of contact of a pair of asperities, and $\left.A_{\mathrm{H}}\right|_{\text {wet }} \approx$ $10^{-21}-\left.A_{\mathrm{H}}\right|_{\mathrm{dry}} \approx 10^{-20} \mathrm{~J}$ is the Hamaker constant with the lower value representing the diminishing interaction energy through water.

At short range $(<0.2 \mathrm{~nm})$, the van der Waals force becomes repulsive [41]. In a wet ultra-thin contact of the type described here, due to very small interfacial energy, the Hamaker constant is further reduced by nearly an order of magnitude (see above). This means

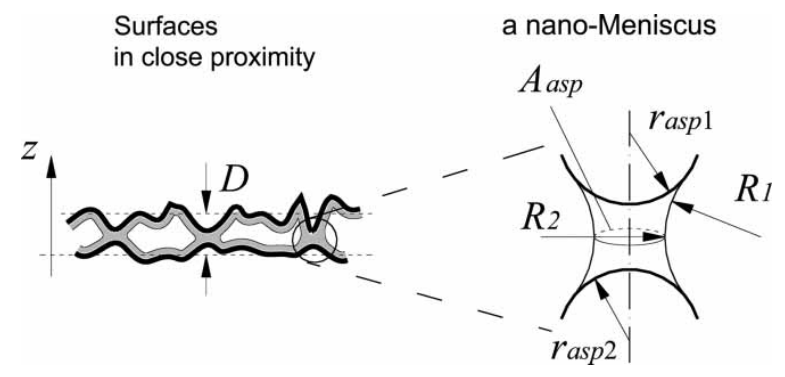

Fig. 4 Nanoscale conjunctions with geometry of a nanomeniscus 
that the density of the water molecules near the surfaces would be expected to undergo an oscillatory behaviour, which would give rise to a solvation-type effect $[\mathbf{2 3}, \mathbf{2 4}]$. When the surfaces are considered to be rough compared with the molecular size of the liquid medium, the solvation effect is dominated by a hydrophobic monotonic behaviour. It is surmised that this hydrophobic behaviour can account for van der Waals repulsive interaction at short range under wet conditions [41]. This monotonic hydrophobic solvation behaviour is referred as hydration (section 5.3).

If there are $N$ asperities per unit area, only $n$ of these would produce van der Waals interaction on a surface area $S$. Thus, using the method proposed by Teodorescu and Rahnejat [42], the force is

$$
\begin{aligned}
F_{\mathrm{W}}= & S \frac{N}{\sigma_{\mathrm{p}} \sqrt{2 \pi}}[\underbrace{\int_{0}^{D-h_{\mathrm{m}}} \mathrm{e}^{-z^{2} / 2 \sigma_{\mathrm{p}}^{2}}\left(-\frac{\left.A_{\mathrm{H}}\right|_{\mathrm{dry}}}{6(D-z)^{2}} R_{\mathrm{e}}\right) \mathrm{d} z}_{\text {for the dry section of the contact }} \\
& +\underbrace{\int_{D-h_{\mathrm{m}}}^{D} \mathrm{e}^{-z^{2} / 2 \sigma_{\mathrm{p}}^{2}}\left(-\frac{\left.A_{\mathrm{H}}\right|_{\text {wet }}}{6(D-z)^{2}} R_{\mathrm{e}}\right) \mathrm{d} z}_{\text {for the wet section of the contact }}]
\end{aligned}
$$

Note that the fully submerged asperities (those between the limits $D$ and $\infty$ ) neither contribute to van der Waals interaction nor to meniscus action.

\subsection{Meniscus action}

Atmospheric moisture condenses to form nanomenisci between the rough surface asperity pairs. The shape of each meniscus (Fig. 4) can be computed by integrating the Young-Laplace equation $p_{\mathrm{o}}-p_{i}+$ $\gamma_{\mathrm{lv}}\left(1 / R_{1}+1 / R_{2}\right)=0$.

The meniscus force for a bridge can be computed as

$$
f_{\mathrm{M}}=-2 \pi R_{\mathrm{e}} \gamma_{\mathrm{lv}}\left(\cos \theta_{1}+\cos \theta_{2}\right)
$$

The contact of a $\beta$-carotene surface with a rough glass is considered here. The contact angle for water with the glass surface is $\theta_{1}=58^{\circ}$ and that with $\beta$-carotene is $\theta_{2}=115^{\circ}[\mathbf{4 3}]$. Note that this obtuse contact angle indicates hydrophobia. This behaviour promotes hydration at close range. The equivalent radius of contact of a pair of asperities for the case investigated (Fig. 4) is $R_{\mathrm{e}}=3.5 \mathrm{~nm}$. The liquid-vapour surface tension is $\gamma_{\mathrm{lv}}=72.78 \mathrm{~mJ}$.

Riedo et al. [31] found the condensation time for one monolayer of water to be around $t_{\mathrm{a}}=25 \mu \mathrm{s}$ and the maximum height, where a meniscus bridge can form after a time $t$, as

$$
h_{\mathrm{m}}(t)=\ln \left(t / t_{\mathrm{a}}\right)\left[\ln \left(p_{\mathrm{s}} / p_{\mathrm{a}}\right) A_{\mathrm{asp}} \rho\right]^{-1}+h_{\mathrm{a}}
$$

If there are $N$ asperities per unit area, only $n$ asperities will form menisci bridges on a surface with an area $S[\mathbf{4 4}]$.

Therefore, the total suction force due to a number of formed menisci is

$$
\begin{aligned}
F_{\mathrm{M}}=n f_{\mathrm{M}}= & -2 \pi R_{\mathrm{e}} \gamma_{\mathrm{lv}}\left(\cos \theta_{1}+\cos \theta_{2}\right) S \frac{N}{\sigma \sqrt{2 \pi}} \\
& \times \int_{D-h_{\mathrm{m}}}^{D} \mathrm{e}^{-z^{2} / 2 \sigma^{2}} \mathrm{~d} z
\end{aligned}
$$

If the entire area is flooded (i.e. all asperities are submerged), then a micromeniscus is formed for the entire contact [43].

\subsection{The hydration force}

The hydrophobic nature of $\beta$-carotene means that a repulsive potential would account for the rupture of water molecules from it. This potential can be considered to be an exponential decay of the form [41]

$$
w_{\mathrm{H}}=2 \gamma_{i} \mathrm{e}^{-(D-z) / \lambda_{0}}
$$

where $\gamma_{i}=10-50 \mathrm{~mJ} / \mathrm{m}^{2}$ and $\lambda_{0}=1.5 \mathrm{~nm}$, which is six times larger than a water molecule $(0.25 \mathrm{~nm})$.

The hydration pressure between a pair of approaching submerged asperities is thus obtained as

$$
p_{\mathrm{Hy}}=\frac{\partial w_{\mathrm{H}}}{\partial(D-z)}=\frac{2 \gamma_{i}}{\lambda_{0}} \mathrm{e}^{-(D-z) / \lambda_{0}}
$$

Thus, for diminishing gaps the force is monotonically increasing. Therefore, for $n$ such asperities

$$
\begin{aligned}
F_{\mathrm{H}}= & A_{\mathrm{asp}} S \frac{2 \gamma_{i}}{\lambda_{0}} \frac{N}{\sigma_{\mathrm{p}} \sqrt{2 \pi}}\left(\int_{D-h_{\mathrm{m}}}^{D} \mathrm{e}^{-z^{2} / 2 \sigma_{\mathrm{p}}^{2}} \mathrm{e}^{-(D-z) / \lambda_{0}} \mathrm{~d} z\right. \\
& \left.+\int_{D}^{\infty} \mathrm{e}^{-z^{2} / 2 \sigma_{\mathrm{p}}^{2}} \mathrm{~d} z\right)
\end{aligned}
$$

Like the solvation effect near solid boundaries, the proposed oscillatory behaviour of hydration is noted only for molecularly smooth surfaces $[\mathbf{2 3}, \mathbf{2 4}, \mathbf{4 1}]$. For surfaces of glass and $\beta$-carotene, (such as spatulae of gecko feet) both being considered as fairly rough when compared with a water molecule, the oscillatory hydration behaviour is rather insignificant.

\subsection{Net contact force}

The net contact force is the combination of the different contributions made as

$$
F=F_{\mathrm{W}}+F_{\mathrm{M}}+F_{\mathrm{H}}
$$




\section{RESULTS AND DISCUSSION}

Data used for the analysis are provided in Appendix 2. The analysis considers two rates of approach of a rough $\beta$-carotene surface from an initial height of $3 \mathrm{~nm}$ towards a rough glass surface, whereas a monolayer of water of $0.25 \mathrm{~nm}$ is initially formed on the latter at an activation time of $25 \mu$ s (Fig. 5). A quasi-static analysis is carried out. It can be seen that equilibrium is reached at a very small height above the logarithmic rising level of the water layer. According to the different rates of descent, little difference is noted between the equilibrium positions reached. With a decreasing gap, the number of dry asperity tip pairs reduces, whereas the number of those forming menisci bridges progressively increases (Fig. 6). In the mean time, some of the counterface asperities become fully submerged in the increasing volume of condensed water. At a given separation (i.e. gap), the wet and fully submerged asperities equate those remaining dry with van der Waals interaction. In the following part, more than half of the surface asperities are wetted and the meniscus effect progresses from one of the multitude of nanomenisci to a larger microscale bridge. It is noticeable that the equilibrium position is reached with a separation greater than the condensed water layer.

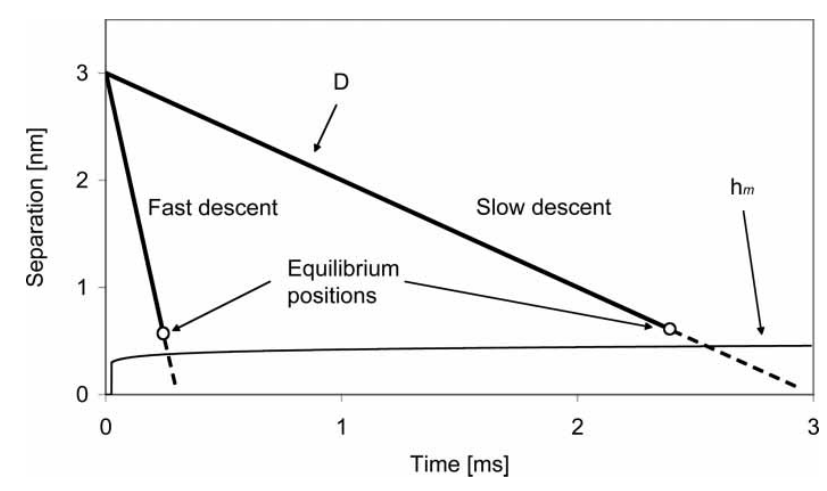

Fig. 5 Quasi-static approach of rough $\beta$-carotene glass conjunction in nanoscale

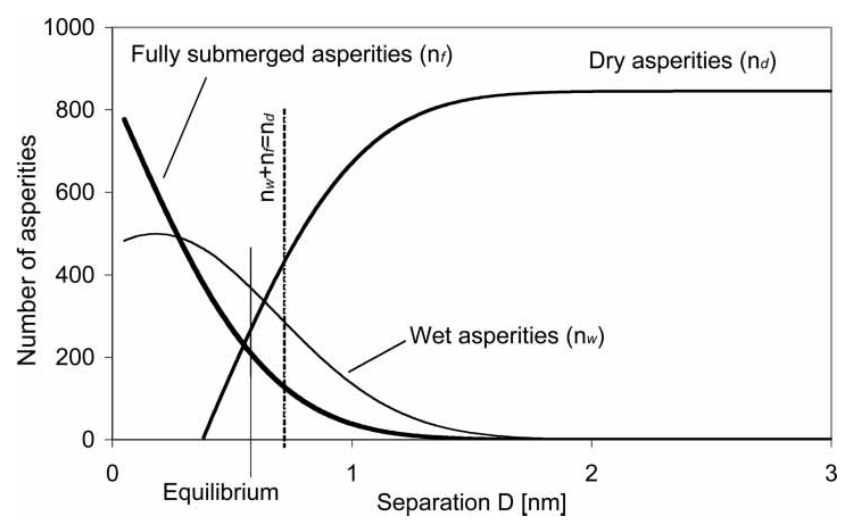

Fig. 6 State of asperity wetness with contact separation
This is possible because most asperities have already been immersed in the thin film of water.

During the approach of surfaces, the number of asperity tips that remain dry, wet, or fully submerged, is continuously changing. Figure 6 shows the state of these asperities as a function of time. It is noticeable that in the centre of the interval, where the separation is at its minimum, the number of dry asperities is very small, resulting in a large microscale meniscus forming over the entire contact.

Figure 7 shows the contribution from each of the acting forces in the conjunction. Referring to the meniscus action, the transition from multiple nanomenisci to one of a microscale bridge is marked by an apparent discontinuity in the graph, which coincides with the aforementioned equality between the number of dry and wet asperities in Fig. 6. In nature, such a transition is likely to occur in a gradual manner.

In Fig. 7, the hydration force increases monotonically as a greater number of asperities submerge into a growing thin film of water. At the same time, a reducing number of dry asperities and the diminishing effect of van der Waals attractive force through wetted surfaces lead to an equilibrium condition marked in Fig. 5. Such equilibria for two different rates of approach are marked in Fig. 5. Following that the most significant changes occur due to the increasing attraction of van der Waals forces through wet asperities and the repulsive hydrophobic potential of hydration in a growing film of water (Fig. 7), the net result of which is shown in Fig. 8.

In practice, it is unlikely for the net force (Fig. 8) to reach $\sim 80 \mathrm{nN}$, as this would lead to buckling of the $\beta$-carotene surface (fibre) or to its detachment during any peeling-off process. To ascertain the limiting net force, an analysis of dynamics of adhesion and peel-off including the compliance of the $\beta$-carotene fibre would be required. Note that the van der Waals attraction force at very close range in wet environments is represented by hydration [41]. Therefore, the appearance of a very large attractive force is

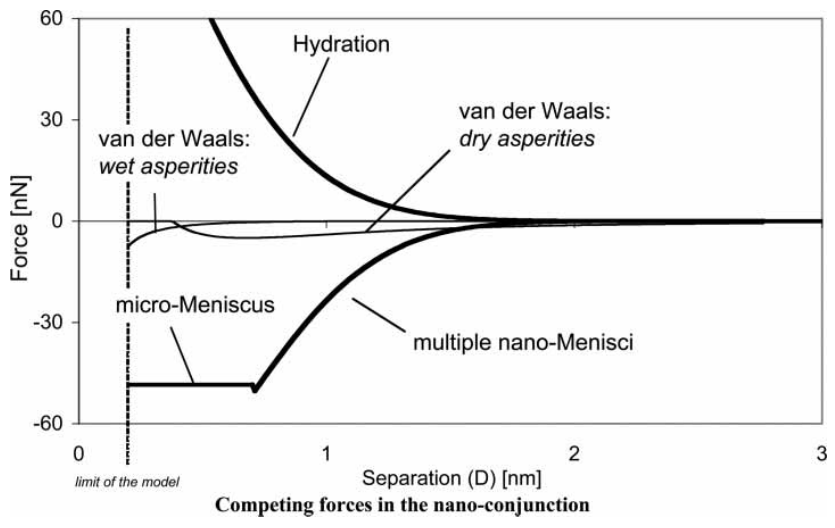

Fig. 7 Competing forces in the nanoconjunction 


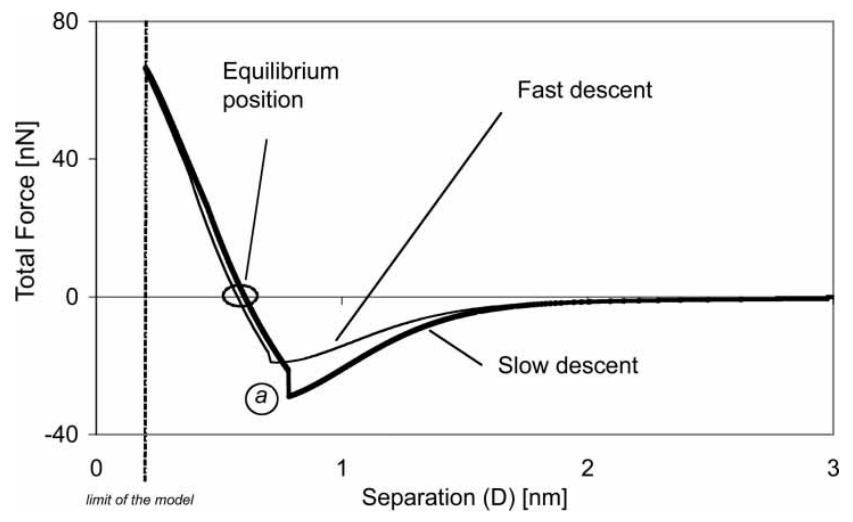

Fig. 8 Net force variation with separation

merely theoretical and not representative of physical conditions. Hence, separations $<0.2 \mathrm{~nm}$ would be unattainable.

The lines in Fig. 8 show the net force between the surfaces. The slight difference in the optimal operating interval for each of the competing kinetics ensures that the total force is either attractive or repulsive. At separations greater than $3 \mathrm{~nm}$, there is no noticeable interaction between the two surfaces. For a diminishing gap, the highest asperities begin forming nanomenisci, and therefore, the attractive force constantly grows with a diminishing gap. At location (a) in Fig. 8, half the contact area is already submerged and all the nanomenisci merge into a single micromeniscus. At even closer proximity, the repulsive hydration action dominates.

\section{CONCLUSIONS}

Newton laid the foundation for the later theories that emerged for the interactions in small scale. These include fluid flow problems expounded through efforts of Navier, Poisson, and Stokes and for narrow conjunctions by Reynolds. Newton's calculus of variation also formed the basis for exposition of deformation theories, among which the classical Hertzian contact mechanics is cited here. When the mechanical nature of continuum gives way to intermolecular and surface energy effects, the direct link to Newton's initial contributions becomes blurred. This occurs in the nanoscale and the minutia beyond it.

\section{REFERENCES}

1 Rahnejat, H. Physics of causality and continuum: questioning nature. Proc. IMechE, Part K: J. Multi-body Dynamics, 2008, 222(K4), 255-264 (this issue).

2 de Coulomb, C. A. Recherches theorique et experimentales sur la force de torsim et l'élasticité des fils de metal, 1784 (L' Académie Royal des Sciences, Paris).
3 Newton, I. Philosophiae naturalis principia mathematica, 1687 (Royal Society, London).

4 Maxwell, J. C. A dynamical theory of the electromagnetic field, 1865 (Royal Society, London).

5 Bohr, N. On the constitution of atoms and molecules (part 1 of 3). Philos. Mag., 1983, 26, 1-25.

6 Bohr, N. On the constitution of atoms and molecules, part II systems containing only a single nucleus. Philos. Mag., 1913, 26, 476-502.

7 Bohr, N. On the constitution of atoms and molecules, part III. Philos. Mag., 1913, 26, 857-875.

8 Einstein, A. Über einen die Erzeugung und Verwandlung des Lichtes betreffenden heuristischen Gesichtspunkt. Ann. Phys. (Leipzig), 1905, 17, 132.

9 Planck, M. Über irreversible Strahlungsvorgänge. Annalen der Physik, 1900, 1, 6.

10 Einstein, A. Die Feldgleichungen der Gravitation. Sitzungsberichte der Königlich Preussischen. Akademie der Wissenschaften, 1915, pp. 844-847.

11 Navier, L. M. H. Memoire sur les lois du mouvement des fluides, vol. 6, 1827 (Memoires de l'Academie Royale des Sciences, Paris).

12 Stokes, G. G. On the theories of the internal friction of fluids in motion, and of the equilibrium and motion of elastic solids. Trans. Camb. Phil. Soc., 1845, 8, 287-305.

13 Poisson, S. D. Memoire sur les equations generales d'equilibre et du mouvement des corps solides elastiques et des fluides. J. de l'Ecole Polytech., 1831, 13, 1.

14 Reynolds, O. On the theory of lubrication and its application to Mr Beauchamp Tower's experiments, including an experimental determination of the viscosity of olive oil. Phil. Trans. R. Soc., 1886, 177, 157-234.

15 Hertz, H. On the contact of elastic solids. J. Reine Angew. Math., 1882, 92, 156-171.

16 Lundberg, G. Elastiche Geruhurng zweir Helbraume. Forschung auf dem Geb. Des Engenieurswesens, 1961, 10(5), 165-174.

17 Leibniz, G. Hypothesis physica nova, 1671 (Hanover).

18 Dowson, D. and Wang, D. An analysis of the normal bouncing of a solid elastic ball on an oily plate. Wear, 1994, 179, 29-37.

19 Al-Samieh, M. F. and Rahnejat, H. Physics of lubricated impact of a sphere on a plate in a narrow continuum to gaps of molecular dimensions. J. Phys. D: Appl. Phys., 2002, 35, 1-16.

20 Eyring, H. Viscosity, plasticity and diffusion as examples of reaction rates. J. Chem. Phys., 1936, 4, 283.

21 Chan, D. Y. C. and Horn, R. G. The drainage of the thin liquid films between solid surfaces. J. Chem. Phys., 1984, 83, 5311-5324.

22 Matsuoka, H. and Kato, T. An ultra-thin liquid film lubrication theory - calculation method of solvation pressure and its applications to EHL problem. Trans. ASME, J. Tribol., 1997, 119, 217-226.

23 Al-Samieh, M. F. and Rahnejat, H. Ultra-thin lubricating films under transient conditions. J. Phys. D: Appl. Phys., 2001, 34, 2610-2621.

24 Teodorescu, M., Balakrishnan, S., and Rahnejat, H. Physics of ultra-thin surface films on molecularly smooth surfaces. Proc. IMechE, Part N: J. Nanoengineering and Nanosystems, 2006, 220(1), 7-19.

25 Kepler, J. Astronomia nova, 1609 (Prague). 
26 Autumn, K., Majidi, C., Groff, R. E., Dittmore, A., and Fearing, R. S. Effective elastic modulus of isolated gecko setal arrays. J. Expl Biol., 2006, 209, 3558-3568.

27 Lee, J., Majidi, C., Schubert, B., and Fearing, R. S. Sliding-induced adhesion of stiff polymer microfiber arrays. I. Macroscale behaviour. J. R. Soc., 2008 (DOI: 10.1098/rsif.2007.1308).

28 Schubert, B., Lee, J., Majidi, C., and Fearing, R. S. Sliding-induced adhesion of stiff polymer microfiber arrays. II. Microscale behaviour. J. R.. Soc., 2008 (DOI: 10.1098/rsif.2007.1309).

29 Teodorescu, M., Theodossiades, S., and Rahnejat, $\mathbf{H}$. Micro-impact dynamics of MEMS gears with rough elastic and SAM protected conjunctions. Tribol. Int. (DOI: 10.1016/j.triboint.2008.05.019).

30 Teodorescu, M., Majidi, C., Fearing, R. S., and Rahnejat, $\mathbf{H}$. Effect of surface roughness on adhesion and friction of microfibers in side contact. In the STLE/ASME International Joint Tribology Conference, Miami, Florida, 20-22 October 2008.

31 Riedo, E., Lévy, F., and Brune, H. Kinetics of capillary condensation in nanoscopic sliding friction. Phys. Rev. Lett., 2002, 88, 185505.

32 Kjellander, R. and Marčelja, S. Perturbation of hydrogen bonding in water near polar surfaces. Chem. Phys. Lett., 1985, 120, 393-396.

33 Attard, P. and Batchelor, M. T. A mechanism for the hydration force demonstrated in a model system. Chem. Phys. Lett., 1988, 149, 206-211.

34 Israelachvili, J. N. and Pashley, R. M. Molecular layering of water at surfaces and origin of repulsive hydration forces. Nature, 1983, 306, 249-250.

35 Pashley, R. Applied colloid and surface chemistry, 2004 (Wiley, New York).

36 Derjaguin, B. V. and Landau, L. Theory of the stability of strongly charged lyophobic sols and the adhesion of strongly charged particles in solutions of electrolytes. Acta Physicochim. (USSR), 1941, 14, 633-662.

37 Verwey, E. J. W. and Overbeek, J. Th. G. Theory of stability of lyophobic coloids, 1948 (Elsevier, Amsterdam).

38 Huber, G. Nanoscale adhesion of individual gecko spatulae explored by atomic force microscopy. PhD Thesis, Universität Stuttgart, Max-Planck-Institut für Metallforschung, 2006.

39 Bhushan, B. and Sayer, A. Surface characterisation and friction of a bio-inspired reversible adhesive tape. Microsyst. Technol., 2007, 13, 71-78.

40 Bocquet, L., Charlaix, E., Ciliberto, S., and Crassous, J. Moisture-induced ageing in granular media and the kinetics of capillarity condensation. Nature, 1998, 396, 735-737.

41 Israelachvili, J. N. Intermolecular and surface forces, 1992 (Academic Press, New York).

42 Teodorescu, M. and Rahnejat, $H$. Dry and wet nanoscale impact dynamics of rough surfaces with or without a self-assembled mono-layer. Proc. IMechE, Part N: J. Nanoengineering and Nanosystems, 2007, 221 (1), 49-58.

43 Huber, G., Mantz, H., Spolenak, R., Mecke, K., Jacobs, K., Gorb, S. N., and Arzt, E. Evidence for capillarity contributions to gecko adhesion from single spatula nanomechanical measurements. Proc. Natl Acad. Sci. USA, 2006, 102(45), 16293-16296.

44 Fuller, K. N. G. and Tabor, D. The effect of surface roughness on the adhesion of elastic solids. Proc. R. Soc., A, 1975, 345(1642), 327-342.

\section{APPENDIX 1}

\section{Notation}

$a$

A

$A_{\text {asp }}$

$A_{\mathrm{H}}$

$c$

E

$E^{\prime}$

$f_{\mathrm{M}}$

$f_{\mathrm{W}}$

F

$F_{\mathrm{H}}$

$F_{\mathrm{M}}$

$F_{\mathrm{W}}$

G

$h$

$\hbar$

$h_{\mathrm{a}}$

$h_{\mathrm{m}}$

K

$l$

$\ell$

L

$m$

$n$

$\boldsymbol{N}$

$N$

$p$

$p_{\mathrm{a}}$

$p_{\mathrm{H}}$

$p_{\text {Hy }}$

$p_{i}$

$p_{\mathrm{m}}$

$p_{\mathrm{o}}$

$p_{\mathrm{s}}$

$q$

q

$r$

$r_{\text {aspl,2 }}$

$r_{1,2}$

$R_{1,2}$

$R_{\mathrm{e}}$

$s$

S

$t$ semi-half-width of contact

area

cross-sectional area of a nanomeniscus bridge

Hamaker constant

speed of light

energy

effective (reduced) elastic modulus

meniscus force per asperity pair

conjunction

van der Waals force per asperity pair

interaction

force

hydration force

total meniscus force in the contact

total van der Waals force in the contact

universal gravitational constant

film thickness

Planck constant

molecular thickness of water

height of meniscus bridge

load-deflection constant of proportionality (stiffness)

Planck length

size of system

length of roller

mass

number of asperities in contact

normal force

total number of asperities

pressure

activation condensation pressure

hydrostatic pressure

hydration pressure

pressure inside a meniscus bridge

mean elastostatic contact pressure

pressure outside a meniscus bridge

saturation pressure

charge

flowrate

radial distance

radii of interacting asperity pairs

radii of contacting rollers

radii of a meniscus

equivalent radius of contacting solids

sliding distance

surface area

time 


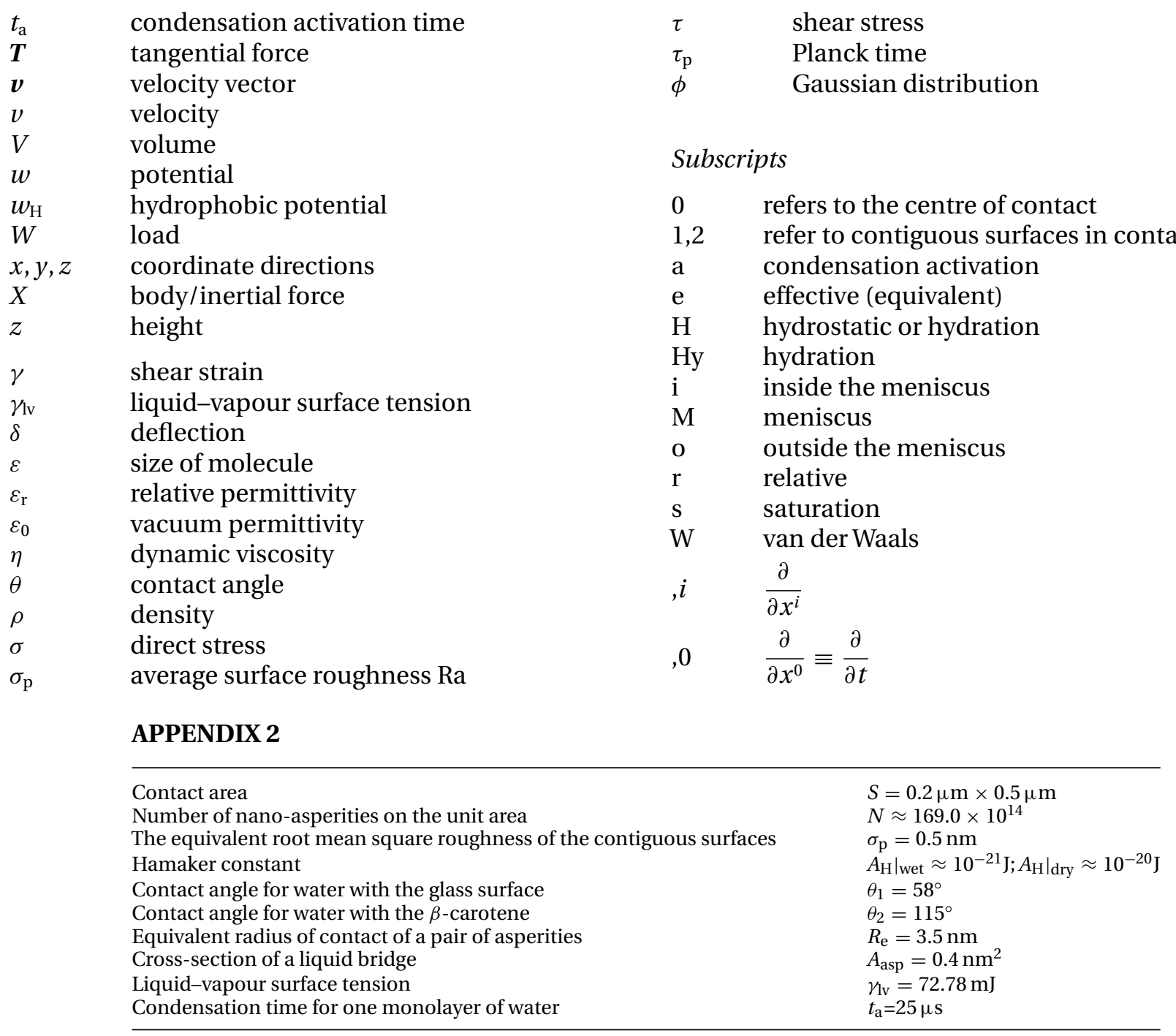

